\title{
Keeping the world at hand: rapid visuomotor processing for hand-object interactions
}

Article

Accepted Version

Makin, T. R., Holmes, N., Brozzoli, C. and Farnè, A. (2012) Keeping the world at hand: rapid visuomotor processing for hand-object interactions. Experimental Brain Research, 219 (4). 421--428. ISSN 0014-4819 doi:

https://doi.org/10.1007/s00221-012-3089-5 Available at https://centaur.reading.ac.uk/28748/

It is advisable to refer to the publisher's version if you intend to cite from the work. See Guidance on citing.

To link to this article DOI: http://dx.doi.org/10.1007/s00221-012-3089-5

Publisher: Springer

All outputs in CentAUR are protected by Intellectual Property Rights law, including copyright law. Copyright and IPR is retained by the creators or other copyright holders. Terms and conditions for use of this material are defined in the End User Agreement.

www.reading.ac.uk/centaur 
Central Archive at the University of Reading

Reading's research outputs online 


\section{TITLE:}

Keeping the world at hand: Rapid visuomotor processing for hand-object interactions

\section{AUTHORS:}

Tamar R. Makin ${ }^{1 *}$, Nicholas P. Holmes ${ }^{2}$, Claudio Brozzoli ${ }^{3}$, Alessandro Farnè ${ }^{4,5}$

\section{AFFILIATIONS:}

1. FMRIB Centre, Oxford University, Oxford, OX3 9DU, UK

2. Centre for Integrative Neuroscience and Neurodynamics, School of Psychology \& Clinical Language Sciences, University of Reading, Reading, RG6 7BE, UK

3. Department of Neuroscience, Brain, Body \& Self lab, Karolinska Institut, SE-17177 Stockholm, Sweden;

4. INSERM U1028, CNRS UMR5292, Lyon Neuroscience Research Centre, ImpAct Team. F69000 Lyon, France;

5. University Claude Bernard Lyon I, F-69000 Lyon, France.

*CORRESPONDING AUTHOR CONTACT DETAILS:

Tamar Makin

Tel : (44) 01865222738

Fax: (44) 01865222717

tmakin@fmrib.ox.ac.uk 


\section{Abstract:}

The existence of hand-centred visual processing has long been established in the macaque premotor cortex. These hand-centred mechanisms have been thought to play some general role in the sensory guidance of movements towards objects, or, more recently, in the sensory guidance of object avoidance movements. We suggest that these hand-centred mechanisms play a specific and prominent role in the rapid selection and control of manual actions following sudden changes in the properties of the objects relevant for hand-object interactions. We discuss recent anatomical and physiological evidence from human and non-human primates, which indicates the existence of rapid processing of visual information for hand-object interactions. This new evidence indicate how several stages of the hierarchical visual processing system may be bypassed, feeding the motor system with hand-related visual inputs within just 70 ms following a sudden event. This time-window is early enough, and this processing rapid enough, to allow the generation and control of rapid handcentred avoidance and acquisitive actions, for aversive and desired objects, respectively.

\section{Keywords:}

Motor control; Peripersonal space; Reference frames; Spatial representation; Superior colliculus. 


\section{Why do we need hand-centred representations of visual space?}

The primate visual system can be described as a series of brain areas whose neuronal activity represents properties of visible objects in the world. One such important property is the location of an object relative to the observer, and this can be represented using one of several different coordinate systems. Perhaps the simplest (and most common) coordinate system is the one based on the retinae of the eyes, in which objects are represented relative to their retinotopic (eye) position. However, in order to interact successfully with a visible object, it is necessary to represent the object's position relative to the observer's body or body part. Given that our hands can move independently from our eyes, the brain needs to integrate information arising in an eye-centred reference frame with information about the current position of the hand relative to the body and to nearby potential target objects.

A well-established solution to this problem involves transforming all information into a common reference frame for the encoding of events. Andersen and colleagues (e.g., Bhattacharyya et al. 2009; Cohen and Andersen 2002) suggested that, within the parietal cortex and via the dorsal visual stream, the represented locations of multisensory cues relevant for actions (e.g., auditory cues arising in head-centred, and proprioceptive cues in limb- or joint-centred frames) are translated into a common eye-centred reference frame. According to this account, action target positions are represented relative to the eyes (or gaze position), and, in its pure form, regardless of the posture of other body parts (Figure 1B). Representations of this type are found throughout the primate visual system, from the retina, via the thalamus and superior colliculus, through the parietal and frontal cortices (Snyder 2000; Buneo and Andersen 2006; Marzocchi et al. 2008), and have repeatedly been demonstrated in humans using both 
imaging and behavioural approaches (Gardner et al. 2008; Crawford et al. 2004). These representations are found so often that they are the principal reference frame for representing visual information in the brain (for an opposing view, see Làdavas 2002).

At the motor end, eye-centred representations of targets would have to be transformed into effector-centred representations, in order to command movements directed towards those targets (Figure 1C). This final transformation between eye- and muscle-centred representations for action may occur in the premotor cortex (for the potential roles of the posterior parietal cortex in coordinate transformations, see Snyder 2000). Within the dorsal premotor cortex (PMd), the firing rate of reach-related neurons is a function of three spatial relationships: between the target and the eye; the target and the hand; and the eye and the hand (Pesaran et al. 2006, 2010). In the ventral premotor cortex (PMv), some neuronal signals have been shown to correspond to the spatial relationship between the hand and the target, independently of eye position (Mushiake et al. 1997; Graziano et al. 1997), or intrinsic movement parameters of individual hand and arm muscles (Kakei et al. 2001). This suggests that the PMv may play a role in the transformation of target location from a visual to a motor frame of reference, relating to broader aspects of skeleto-motor control than previously assigned to it. At this point, it is important to emphasise that, according to the common eye-centred account presented above, while this final stage of eye-to-hand coordinate transformation is necessary for launching the motor behaviour, it is only performed after the motor outcome has been decided on and planned (Snyder 2000). In this respect, hand-centred visual representations are not used for on-going sensory representations, but rather for enabling motor outputs. 
While the scheme of transforming all inputs into a single (eye-centred) representation frame may be common, it may not be the most efficient for controlling a moving body from a signal processing point of view (e.g., Glennerster et al. 2001). This is because sensorimotor transformations come with some costs. First, transformations may add biases and variability to the transformed signals, influencing the flow and quality of information in motor control circuits (McGuire and Sabes 2009; Schlicht and Schrater 2007). Second, transformations take time. In our daily interactions with objects, however, we often need to respond very quickly to unexpected changes in their position or movement, and speed is of paramount importance. Moreover, as we move around, changes in our body position (and particularly our hand-position) will require constant updating and re-calibration of the positions of objects relative to us, in the common reference frame. Given this error, bias, and delay with each sensory-motor transformation, and for each movement of the subject or object, it is appealing to speculate that the brain may possess an alternative, simpler, and more rapid mechanism for processing visual information to generate effectorcentred motor commands to a very tight deadline.

In the following, we will present and discuss anatomical and physiological evidence indicating the existence of rapid processing of visual information for hand-object interactions. We propose that the neural mechanisms previously implicated with hand-centred processing of objects in near space (peripersonal space, see Makin et al. 2008), in particular those within the PMv (e.g., Rizzolatti et al. 1981; Graziano et al. 1994; see also Caggiano et al. 2009), may play a specific role in the rapid processing of visual information for the on-line control of actions. 


\section{Anatomical pathways for rapid processing of visual information}

Which brain circuits might rapidly mediate the flow of visual information to the motor system? Most retinal output projects to the striate cortex via the thalamic lateral geniculate nucleus (LGN), and ascends up the visual hierarchy in an eyecentred fashion (Maunsell and Newsome 1982). A small proportion of the retinal output bypasses this major pathway and projects instead to the superior colliculus in the midbrain (Perry and Cowey 1984; see also Sincich et al. 2004, for an alternative pathway directly connecting the LGN to the middle temporal visual area, MT). This collicular pathway may be involved in the rapid processing of visual information for action: Lyon and colleagues (Lyon et al. 2010) used a rabies virus in macaque monkeys in order to track transynaptic connections projecting from the superior colliculus towards the cerebral cortex. They found that areas within the visual cortical dorsal stream (i.e., the third retinotopic visual area, V3a, and MT) receive disynaptic projections from the superior colliculus via an inferior pulvinar relay (see Berman and Wurtz 2010, for complementary physiological results). No evidence was found for similar projections from the superior colliculus to the visual ventral stream areas. This exciting new finding, summarized here in Figure 2, places the PMv within just five synapses from the retina (via the superior colliculus, the inferior pulvinar, MT and the ventral intraparietal area (VIP), see Kaas and Lyon 2007; Lewis and Van Essen 2000). This "express" route should be able to transfer visual information to the motor cortex within approximately $70-80 \mathrm{~ms}$ (see Pettersson et al. 1997 for comments on synaptic relay times during the online visual control of movement in cats). Lyon and colleagues assigned the collicular-to-dorsal visual stream route with the functional role of processing rapidly-moving visual stimuli. Here we extend this suggestion, and hypothesize that this route might be directly responsible for the 
rapid, on-line updating of changing visual information with respect to hand position that is crucial for the dynamic control of action (Cisek and Kalaska 2010; see also Stuphorn et al. 2000; Reyes-Puerta et al. 2010, for evidence for reachrelated visual coding in the intermediate layers of the superior colliculus).

\section{Physiological evidence for the rapid on-line control of action}

These anatomical data from monkeys (Lyon et al. 2010) coincide with human neurophysiological data from experiments in our laboratory, demonstrating rapid processing of visual information relevant for motor control. In our study (Makin et al. 2009), participants performed a simple button-press motor-response with the right index finger, while a task-irrelevant three-dimensional ball suddenly fell just above the participants' responding hand. Using single-pulse transcranial magnetic stimulation (TMS) over the contralateral primary motor cortex, we found that the sudden appearance of this potentially threatening visual stimulus was associated with a reduction in corticospinal excitability at the very early and specific time window of 70-to-80ms following its appearance (see Evarts 1974, for comparable response latencies in macaque M1 neurons, with respect to visual feedback for force application). We interpreted this inhibition as reflecting the proactive suppression of an automatic avoidance-related response, during the execution of the task-related response. Indeed, when the two motor behaviours (the avoidance and the task-related responses) were uncoupled, the approaching ball had an opposite, facilitatory effect on corticospinal excitability (Figure 3A). Importantly, the rapid inhibition of corticospinal excitability was predominantly hand-centred, depending most upon the distance of the ball from the hand, regardless of the locations of both visual fixation and covert spatial attention relative to the ball and hand. 
This ability to inhibit one movement while concurrently executing another (selective inhibition) is crucial for flexible motor behaviour (Coxon et al. 2007). Such inhibition can be effective not only to suppress undesirable movements after they have been initiated, but also proactively, before any muscle response is released (Boulinguez et al. 2008). The rapid processing of visual information during action execution is therefore a pre-requisite for such proactive inhibition. Since the hierarchical processing of information in the visual cortex is relatively slow (latencies of neurons in macaque secondary visual cortex (V2), for example, span between $56-117 \mathrm{~ms}$, Schmolesky et al. 1998), it is likely that the rapid reprogramming in response to visual information that we demonstrated in our study is mediated via more direct pathways (e.g., area MT, which exhibits latencies as early as $49 \mathrm{~ms}$ ), possibly including the rapid subcortical route identified by Lyon and colleagues (Lyon et al. 2010). Indeed, the rapid handcentred modulations in our study fit well with the estimated time for processing visual information via the SC in humans, based on recordings in the cat brain (Pettersson et al. 1997). Our findings also correspond well with kinematic and electromyographic findings for early movement corrections following unexpected changes in target positions, just under $100 \mathrm{~ms}$ following the perturbation (Paulignan et al. 1991; Farnè et al. 2003; Pruszynski et al. 2008). This timing fits very well with our findings, assuming a $\sim 25 \mathrm{~ms}$ conduction time between the primary motor cortex and the intrinsic hand muscles.

While single-pulse TMS allowed us accurately to infer the time-line of handcentred representations of visual information, it did not provide us with any evidence regarding the brain mechanisms enabling such processing. A recent study directly links the rapid selection of motor responses following changes in 
visual information with the PMv. Buch and colleagues (Buch et al. 2010) used paired-pulse TMS in order to examine the involvement of the right PMv in the reprogramming of grasp apertures when a target cylinder unexpectedly changed in size (from 15 to $65 \mathrm{~mm}$ or vice versa), as compared to when the movement was executed towards the originally planned target size. The authors found that PMv facilitated corticospinal excitability prior to and following the onset of the planned movement (see also Koch et al. 2006; O'Shea et al. 2007; Davare et al. 2006 , for similar results). When the planned movement had to be reprogrammed following a change in the target, however, the PMv now inhibited corticospinal excitability (Figure 3B). Crucially, this inhibitory effect emerged as early as $75 \mathrm{~ms}$ following the change in visual information, and after the original reaching movement had been launched.

Contrary to our inhibitory effect, which was confined to a narrow time window of less than $20 \mathrm{~ms}$, the effect reported by Buch and colleagues remained significant until at least $100 \mathrm{~ms}$ following the switch. This difference between the two studies might reflect the transient nature of the conflict between the avoidance and simple reaction time responses in our design, as compared to the on-going reaching and grasping movement that Buch and colleagues studied. Indeed, it is important to note that the mechanisms underlying these two types of motor behaviour (avoidance vs. grasping) are most likely different. However, both studies examined representations of changing visual information that are crucial for rapid decision making during motor control. The generation and control of both avoidance and grasping movements in a dynamic environment require repositioning of the hand in space, and coordination between proximal and distal muscles (for example, changes in object size will require re-programming of the transport phase, Castiello et al. 1993). In our study, the relevant visual 
information was a ball, approaching the subject at high velocity $(\sim 370 \mathrm{~cm} / \mathrm{s})$. In the study by Buch and colleagues, the target object suddenly changed in size by $50 \mathrm{~mm}$, which was brought about via a change in illumination in a darkened room. Such transient, high-contrast, and high-luminance visual events would activate many visual pathways, likely including those also responsive to rapidly moving objects. Given the similarity in onset times of the visual modulation of motor excitability in these two reports, we propose that the mechanisms responsible for the rapid reprogramming of hand and arm movements based on visual perturbations, partly rely on the same neural pathways that give rise to rapid hand-centred representations of space when examined neurophysiologically. We expand upon this notion in the next sections.

\section{Hand centred representations in PMv}

The premotor cortex has often been implicated with eye-to-hand coordinate transformation (Pesaran et al. 2006, 2010; Kakei et al. 2001; Mushiake et al. 1997). The most striking demonstrations of hand-centred representation of visual information have been found in bimodal visual-tactile neurons in the macaque PMv (Graziano et al. 1994, 1997; Graziano 1999; Rizzolatti et al. 1981, see Figure 1B). In these bimodal neurons, the visual and tactile receptive fields generally overlap. This characteristic ensures that the neurons respond preferentially to visual stimuli near the tactile receptive field on the hand, arm, shoulders, neck, or face. Evidence for visual-tactile integration is not exclusive to the hand (e.g., Farne et al., 2005), or to the representation of visual space (e.g. Serino et al., 2011). However, as both vision and the hands play a dominant role in humans for interactions with objects, we will focus our discussion on neurons 
representing visual information around the hands and arms. Furthermore, bodypart centred multisensory representations are not restricted to the PMv, and have also been shown in the posterior parietal cortex, particularly in area VIP (Cooke et al., 2003), which connects MT with PMv directly (ref from matelli luppino here see Figure 2). However, since hand-centred visual representations are most commonly found in the PMv, we will restrict our discussion to this particular area, without considering the relative contributions of the posterior parietal cortex. The body-part centred multisensory neurons were proposed to provide a general solution to the problem of visuomotor integration (Graziano and Gross 1998), and have stimulated much research in humans (for review, see Brozzoli et al. 2011). The accepted view over the years has been that visual hand-centred mechanisms should play some general role in the sensory guidance of movements towards objects (Rizzolatti 1987; Graziano and Gross 1998; Fogassi and Luppino 2005), or, more recently, in object avoidance responses (Graziano et al. 2002; Cooke et al. 2003), but their specific role has not been determined.

The response properties of macaque PMv hand-centred neurons are similar to the hand-centred modulations of motor excitability by visual stimuli that we found recently using TMS (Makin et al. 2009): In both cases, modulations in motor excitability varied with the distance of the object from the hand, independently of the retinal position of the visual stimulus. Moreover, hand-centred modulation was specific for three-dimensional objects approaching the hand. Although comparisons between data arising from monkeys and humans, and using such different methods, should be made with caution, given the spatial specificity of the above responses with respect to visual events, and given the evidence for the involvement of PMv in rapid visuomotor response selection (Buch et al. 2010), we suggest that these hand-centred mechanisms play a specific and 
prominent role in the rapid selection and control of manual actions. Moreover, as the responses of these hand-centred PMv neurons are generally strongest for real, three dimensional, moving stimuli, we suggest that hand-centred coding occurs predominantly to update the motor system about unexpected changes in the visual properties of objects which are relevant for hand actions during the online control of action.

It is important to emphasise that we do not assign an exclusive role of the PMv in the representation of visual information for the rapid on-line control of movement. Indeed, Cisek and Kalaska (2010) noted that the continuous and parallel processes critical for hand-object interactions appear as two waves of activation: an early wave $(<100 \mathrm{~ms})$, crudely specifying a "menu" of options; and a second wave that selects among the different available options approximately 120 150ms after visual stimulus onset (Cisek and Kalaska 2010). We suggest that when a rapid motor decision is required (such as when an initiated movement needs to be corrected or aborted, due to unexpected changes in the object's properties), transient hand-centred visual information, made available within a short time frame (potentially due to a specialized pathway), will be utilized (however, see Davare et al. 2006, for rapid modulation of PMv responses? during the delayed execution of a pre-planned movement). Eye-centred representations for action, which during hand-movements will necessitate recurrent transformations (from hand-centred to eye-centred, and then back again to hand-centred), are likely to dominate the later wave. We are currently not aware of direct evidence using electrophysiology to support the role of the PMv in rapid hand-centred visual representations during hand-object interactions. An indirect finding was reported by Cooke and Graziano (2003), which showed startle-related EMG activity occurring as early as $70 \mathrm{~ms}$ after stimulus onset. This 
EMG activity was comparable to the muscle responses that were artificially evoked by electrical macro-stimulation of bimodal regions of the premotor cortex (Graziano et al. 2002). We hope that our suggestions will motivate researchers to design paradigms involving hand-movements while studying hand-centred representations in PMv.

\section{Concluding remarks}

A quick review of the literature reveals that the most dominant representation for visually guided hand movements is an eye-centred one. Moreover, the theoretical framework that promotes the primary role of eye-centred representations in hand-object interactions willingly engulfs evidence showing hand-centred processing of the position of objects in the motor system, as a final stage in the perception-to-action pathway (Snyder 2000).

We suggest that a short and rapid pathway from the retina to the motor cortex exists to update hand-relevant visual information for the dynamic control of handobject interactions. According to our framework, sudden or unexpected changes in the location of objects relative to the hand will be rapidly transmitted to the PMv via a rapid subcortical route involving the superior colliculus. This relatively "crude" visual information will be integrated with sensory information regarding current estimates of hand position in the PMv, resulting in hand-centred responses in bimodal neurons with overlapping visual and tactile receptive fields (Graziano et al. 1999). This hand-centred information will then be used to abort or inhibit preplanned movements that become irrelevant, or to facilitate newly 
emerging movements. Such a mechanism might allow for the rapid selection of appropriate actions, for example when the position of a target object unexpectedly changes (e.g., a glass that falls from the shelf as we fumble in reaching for it) or when we are unexpectedly required to avoid an approaching object (e.g., realizing that we are, in fact, unable to catch the falling glass, we instead try to avoid injury). In accordance with the model proposed by Cisek and Kalaska (2010), we emphasise the role of this rapid mechanism in an unstable environment. This is because under predictable conditions, hand-centred representation could be out-weighed by the eye-centred mechanisms, which provide more accurate visuospatial information relating to the positions of the object and the hand.

Cooke and Graziano have previously proposed a similar notion, advocating for the involvement of bimodal neurons in the PMv (as well as the ventral intraparietal area) in the coding of avoidance responses (Cooke and Graziano 2003; Cooke et al. 2003; Graziano et al. 2002). Our proposal extends this framework in several ways: First, we argue that the same coding occurs during the rapid selection of responses both towards objects (reaching and grasping) and away from objects (avoidance). The same neural mechanisms and pathways may sustain both types of hand-object interaction. Second, according to our account, hand-centred processing of objects in this pathway can only occur (or at least, predominantly occurs) during sudden changes in the properties most relevant for hand-object interactions, such as the spatial properties of the object. In this sense, therefore, there is no need for a continuous, online hand-centred representation of peripersonal space. Instead, hand-centred visual representations of objects near or approaching the hand are dynamically formed only whenever they become relevant for our actions and interactions with the 
world. We therefore suggest that these mechanisms specialize in the rapid updating of relevant visual information during response-selection and the on-line control of action. We believe that future studies should place an emphasis on the precise timing of visuomotor transformations, as well as multisensory interactions during action (see, for example, Brozzoli et al, 2009, 2010), as important criteria for determining which are the underlying neural mechanisms for hand-centred representations. 


\section{References}

Berman RA, Wurtz RH (2010) Functional identification of a pulvinar path from superior colliculus to cortical area MT. J Neurosci 30:6342-6354

Bhattacharyya R, Musallam S, Andersen RA (2009) Parietal reach region encodes reach depth using retinal disparity and vergence angle signals. $J$ Neurophysiol 102:805-816

Boulinguez P, Jaffard M, Granjon L, Benraiss A (2008) Warning signals induce automatic EMG activations and proactive volitional inhibition: Evidence from analysis of error distribution in simple RT. J Neurophysiol 99:1572-1578

Brozzoli C, Makin T, Cardinali L, Holmes NP, Farnè A (2011) Peripersonal Space: A multisensory interface for body-object interactions. In: Murray MM, Wallace MT. Frontiers in the Neural Bases of Multisensory Processes. Lausanne (Switzerland): CIBM

Buch ER, Mars RB, Boorman ED, Rushworth MFS (2010) A network centered on ventral premotor cortex exerts both facilitatory and inhibitory control over primary motor cortex during action reprogramming. J Neurosci 30:13951401

Buneo CA, Andersen RA (2006) The posterior parietal cortex: sensorimotor interface for the planning and online control of visually guided movements. Neuropsychologia 44:2594-2606

Caggiano V, Fogassi L, Rizzolatti G, Thier P, Casile A (2009) Mirror neurons differentially encode the peripersonal and extrapersonal space of monkeys. Science 324:403-406 
Castiello U, Bennett KMB, Stelmach GE (1993) Reach to grasp: The natural response to perturbation of object size. Exp Brain Res 94:163-178

Cisek P, Kalaska JF (2010) Neural mechanisms for interacting with a world full of action choices. Annu Rev Neurosci 33:269-298.

Cohen YE, Andersen RA (2002) A common reference frame for movement plans in the posterior parietal cortex. Nature Rev Neurosci 3:553-562

Cooke DF, Graziano MS (2003) Defensive movements evoked by air puff in monkeys. J Neurophysiol 90:3317-3329.

Cooke DF, Taylor CSR, Moore T, Graziano MSA (2003) Complex movements evoked by microstimulation of the ventral intraparietal area. Proc Natl Acad Sci U S A100:6163-6168

Coxon JP, Stinear CM, Byblow WD (2007) Selective inhibition of movement. J Neurophysiol 97:2480-2489

Crawford JD, Medendorp WP, Marotta JJ (2004) Spatial transformations for eyehand coordination. J Neurophysiol 92:10-19.

Davare M, Andres M, Cosnard G, Thonnard JL, Olivier E (2006) Dissociating the role of ventral and dorsal premotor cortex in precision grasping. J Neurosci 26:2260-2268

Evarts EV (1974) Precentral and postcentral cortical activity in association with visually triggered movement. J Neurophysiol 37:373-381.

Farnè A, Demattè ML, Ladavas E (2005) Neuropsychological evidence of modular organization of the near peripersonal space. Neurology 13:17541758.

Farnè A, Roy AC, Paulignan Y, Rode G, Rossetti Y, Boisson D, Jeannerod M (2003) Visuo-motor control of the ipsilateral hand: evidence from right braindamaged patients. Neuropsychologia. 41:739-757. 
Fogassi L, Luppino G (2005) Motor functions of the parietal lobe. Curr Opin Neurobiol15:626-631

Gardner JL, Merriam EP, Movshon JA, Heeger DJ (2008) Maps of visual space in human occipital cortex are retinotopic, not spatiotopic. J Neurosci 28:3988-3999.

Glennerster A, Hansard ME, Fitzgibbon AW (2001) Fixation could simplify, not complicate, the interpretation of retinal flow. Vision Res 41:815-834

Graziano MSA (1999) Where is my arm? The relative role of vision and proprioception in the neuronal representation of limb position. Proc Natl Acad Sci U S A 96:10418-10421

Graziano MS (2006) Progress in understanding spatial coordinate systems in the primate brain. Neuron 51:7-9

Graziano MSA, Hu XT, Gross CG (1997) Visuospatial properties of ventral premotor cortex. J Neurophysiol 77:2268-2292

Graziano MS, Gross CG (1998) Spatial maps for the control of movement. Curr Opin Neurobiol 8:195-201

Graziano MSA, Taylor CSR, Moore T (2002) Complex movements evoked by microstimulation of precentral cortex. Neuron 34:841-851

Graziano MSA, Yap GS, Gross CG (1994) Coding of visual space by premotor neurons. Science 266:1054-1057

Kaas JH, Lyon DC (2007) Pulvinar contributions to the dorsal and ventral streams of visual processing in primates. Brain Res Rev 55:285-96

Kakei S, Hoffman DS, Strick PL (2001) Direction of action is represented in the ventral premotor cortex. Nat Neurosci 10:1020-1025.

Koch G, Franca M, Del Olmo MF, Cheeran BJ, Milton R, Alvarez Sauco M, Rothwell JC (2006) Time course of functional connectivity between dorsal 
premotor and contralateral motor cortex during movement selection. $\mathrm{J}$ Neurosci 26:7452-7459

La'davas E (2002) Functional and dynamic properties of visual peripersonal space. Trends Cogn Sci 6:17-22.

Lewis JW, Van Essen DC (2000) Corticocortical connections of visual, sensorimotor, and multimodal processing areas in the parietal lobe of the macaque monkey. J Comput Neurosci 428:112-137

Lyon DC, Nassi JJ, Callaway EM (2010) A disynaptic relay from superior colliculus to dorsal stream visual cortex in macaque monkey. Neuron $65: 270-279$

Makin TR, Holmes NP, Brozzoli C, Rossetti YRC, Farnè A (2009) Coding of visual space during motor preparation: Approaching objects rapidly modulate corticospinal excitability in hand-centred coordinates. J Neurosci 29:11841-11851

Makin TR, Holmes NP, Ehrsson HH (2008) On the other hand: dummy hands and peripersonal space. Behav Brain Res 191:1-10

Marzocchi N, Breveglieri R, Galletti C, Fattori P (2008) Reaching activity in parietal area $\mathrm{V} 6 \mathrm{~A}$ of macaque: eye influence on arm activity or retinocentric coding of reaching movements? Eur J Neurosci. 27:775-789

McGuire LMM, Sabes PN (2009). Sensory transformations and the use of multiple reference frames for reach planning. Nature Neurosci 12:10561061

Maunsell JH, Newsome WT (1978) Visual processing in monkey extrastriate cortex. Annual Rev Neurosci 10:363-364

Mushiake H, Tanatsugu Y, Tanji J (1997) Neuronal activity in the ventral part of premotor cortex during target-reach movement is modulated by direction of gaze. J Neurophysiol 78:567-571. 
O'Shea J, Sebastian C, Boorman ED, Johansen-Berg H, Rushworth MFS (2007) Functional specificity of human premotor-motor cortical interactions during action selection. Eur J Neurosci 26:2085-2095

Paulignan Y, MacKenzie C, Marteniuk R, Jeannerod M (1991) Selective perturbation of visual input during prehension movements. 1. The effects of changing object position. Exp Brain Res 83:502-512

Perry VH, Cowey A (1984) Retinal ganglion cells that project to the superior colliculus and pretectum in the macaque monkey. Neurosci 12:1125-1137

Pesaran B, Nelson MJ, Andersen RA (2006) Dorsal premotor neurons encode the relative position of the hand, eye, and goal during reach planning. Neuron 51:125-134

Pesaran B, Nelson MJ, Andersen RA (2010) A relative position code for saccades in dorsal premotor cortex. J Neurosci 30:6527-6537

Pettersson LG, Lundberg A, Alstermark B, Isa T, Tantisira B (1997) Effect of spinal cord lesions on forelimb target-reaching and on visually guided switching of target-reaching in the cat. Neurosci Res 29:241-256

Pruszynski JA, Kurtzer I, Scott SH (2008) Rapid motor responses are appropriately tuned to the metrics of a visuo-spatial task. J Neurophysiol 100:224 -238

Reyes-Puerta V, Philipp R, Lindner W, Hoffmann KP (2010) Role of the rostral superior colliculus in gaze anchoring during reach movements. $\mathrm{J}$ Neurophysiol 103:3153-3166

Rizzolatti G (1987) Functional organization of inferior area 6. Ciba Found Symp 132:171-186

Rizzolatti G, Luppino G, Matelli M (1998) The organization of the cortical motor system: new concepts. Electroencephalogr Clin Neurophysiol 106:283-296 
Rizzolatti G, Scandolara C, Matelli M, Gentilucci M (1981) Afferent properties of periarcuate neurons in macque monkeys. II. Visual responses. Behav Brain Res 2:147-163

Schlicht EJ, Schrater PR (2007) Impact of coordinate transformation uncertainty on human sensorimotor control. J Neurophysiol 97:4203-4214

Schmolesky MT, Wang Y, Hanes DP, Thompson KG, Leutgeb S, Schall JD, Leventhal AG (1998) Signal timing across the macaque visual system. J Neurophysiol 79:3272-3278

Serino A, Canzoneri E, Avenanti A. (2011) Fronto-parietal areas necessary for a multisensory representation of peripersonal space in humans: an rTMS study. J Cogn Neurosci 23:2956-2967

Sincich LC, Park KF, Wohlgemuth MJ, Horton JC (2004) Bypassing V1: a direct geniculate input to area MT. Nat Neurosci 7:1123-1128

Snyder LH (2000) Coordinate transformations for eye and arm movements in the brain. Curr Opin Neurobiol 10:747-754.

Stuphorn V, Bauswein E, Hoffmann KP (2000) Neurons in the primate superior colliculus coding for arm movements in gaze-related coordinates. $\mathrm{J}$ Neurophysiol 83:1283-1299. 
Figure 1. Eye- and hand-centred spatial representations in the primate brain. Response patterns of illustrative neurons which are specifically modulated by changes in the fixation position (with respect to reaching targets B), and by changes in hand position (with respect to an approaching object, C). In both paradigms, monkeys were trained to maintain fixation in one of three targets (indicated by crosses in $\mathbf{A}$ ) and place their hand in one of three positions (as indicated by the sketch in A). In B, responses were recorded in the parietal reach region (PRR, shown in $\mathbf{A}$ ), while monkeys were performing delayed reaching movements to each of four different targets (indicated by the gray circles in $\mathbf{A}$, corresponding to the four panels in each row). In C, responses were recorded in the ventral premotor cortex (PMv, shown in $\mathbf{A})$, while three-dimensional objects were approaching the monkey from four different trajectories (indicated by the gray arrows in A, corresponding to the four panels in each row). Initial hand and fixation positions are shown in the left side of each row. In both studies, hand and eye positions were independently manipulated (upper and lower two rows, 
respectively). In B, the peak response of the neuron shifted when the initial eye position was varied, but not when the initial hand position was varied. In $\mathbf{C}$, the peak response of the neuron shifted when the initial hand position was varied, but not when the initial eye position was varied. Figure 1B was modified from Cohen and Andersen (2002). Figure $1 \mathrm{~A}$ and $1 \mathrm{C}$ was modified from Graziano et al. (1997).

Figure 2. Fast route from visual input to motor output.

Most retinal output projects to the striate cortex via the thalamic lateral geniculate nucleus (LGN), and ascends up to the motor cortex via the dorsal and ventral pathways (gray arrows). A small proportion of the retinal output bypasses this major pathway and projects instead to the superior colliculus (SC) in the midbrain (gray arrows). Information from the SC is transferred to the middle temporal visual area (MT) via disynaptic projections. This places the premotor cortex (PMv) within just five synapses from the retina (via the SC, the inferior pulvinar, MT and the ventral portion of intraparietal sulcus (VIP). By effectively bypassing the visual processing hierarchy, visual information mediated via this pathway may therefore become available for rapid online control of action.

Figure 3. Rapid modulation of TMS-facilitated corticospinal excitability during the on-line control of pre-programmed and modified movements. A. Modulations in corticospinal excitability, measured using single TMS pulses over M1, during unexpected appearance of distractor 3D objects, approaching the participants. When participants responded to the task irrelevant objects (identified by a voluntary muscle twitch of the approached hand), corticospinal excitability was specifically enhanced when the object was approaching near the hand, resulting in hand-centred excitation, 70ms following the appearance of the object (left). 
When the participants were pre-engaged in a reaction time task, the unexpected appearance of the objects approaching the hand caused reduced corticospinal excitability, resulting in hand-centred suppression, $80 \mathrm{~ms}$ following the object appearance. A. Modulations in corticospinal excitability, measured using ventral premotor cortex (PMv) and primary motor cortex (M1) paired-pulse TMS, during the performance of reaching movements to objects. When the same object was maintained throughout the execution of the movement, PMv pulses resulted in increased corticospinal excitability $75 \mathrm{~ms}$ following the onset of the movement (left). When the target object was switched during movement execution, PMv pulses suppressed corticospinal excitability as early as $75 \mathrm{~ms}$ following the switch (which was time locked to the movement onset, right). Figure $3 \mathrm{~A}$ was modified from Makin et al. (2009). Figure 3B was modified from Buch et al. (2010). 